Conditions cadres pour l'exercice de la médecine carcérale: prise de position de la Commission Centrale d'Ethique de l'ASSM

\title{
Autorité de l'Etat et éthique médicale
}

Commission Centrale d'Ethique de l'Académie Suisse des Sciences Médicales (ASSM)
Correspondance: Académie Suisse des Sciences Médicales (ASSM) Petersplatz 13 CH-4051 Bâle mail[at]samw.ch
En 2010, la prise en charge médicale d'un détenu en grève de la faim et la participation de médecins aux renvois forcés avaient défrayé la chronique; parallèlement, l'Académie Suisse des Sciences Médicales (ASSM) s'est penchée sur ce sujet. Le Tribunal fédéral avait alors stipulé que l'autorité d'exécution devait ordonner une alimentation forcée, s'il s'agit du seul moyen d'éviter des lésions irréversibles ou la mort du détenu. Toutefois, basés sur l'éthique professionnelle et notamment sur les directives de l'ASSM «Exercice de la médecine auprès de personnes détenues», les médecins concernés avaient refusé de procéder à une alimentation forcée.

L'ASSM a saisi cette occasion pour vérifier l'actualité et la praticabilité de ses directives médicoéthiques relatives à «L'exercice de la médecine auprès de personnes détenues» qui datent de 2002. Cette analyse a montré que ces directives, rédigées sur la base de documents internationaux mondialement reconnus, restent valables. Elles sont ancrées dans l'éthique médicale et dans le droit en vigueur. Les expériences des médecins pénitentiaires montrent cependant que les principes éthiques énoncés dans les directives sont encore insuffisamment appliqués dans l'exécution des peines et des mesures. Le but de cette prise de position est de rappeler les principes fondamentaux de la médecine carcérale et de formuler des recommandations pour améliorer leur mise en œuvre.

\section{Equivalence des soins}

La personne détenue a droit à des soins équivalents à ceux dont bénéficie la population générale (directives de l'ASSM, chapitre 5).

Sur la base du principe de l'équivalence des soins, ancrée non seulement dans le droit national [1] mais également dans des directives et recommandations internationales [2], les personnes détenues ont droit à des soins équivalents à ceux dont bénéficient les autres patients. Ils peuvent prétendre à toutes les mesures préventives, diagnostiques, thérapeutiques ou de soins correspondant au standard médical. Le droit à l'équivalence des soins ne se limite pas à l'accès aux soins dans toute leur étendue, mais comprend aussi le respect des droits du patient comme le droit à l'autodétermination et à l'information ou le droit au respect de la confidentialité.

En pratique cependant, différents facteurs sont susceptibles de mettre en péril l'équivalence des soins:
- Il est trop peu tenu compte du fait que les personnes détenues présentent une prévalence plus élevée de maladies infectieuses, d'addictions et de troubles psychiques [3] et que des efforts spécifiques sont nécessaires pour garantir une prise en charge médicale adéquate de ces patients.

- Il n'y a pas assez de professionnels qualifiés. Pour des raisons d'organisation, le personnel de surveillance doit souvent se charger de tâches médicales (par ex. distribution de médicaments), ce qui peut notamment transgresser le principe de la confidentialité (protection du secret professionnel).

- Tous les spécialistes impliqués dans la prise en charge médicale (médecins, soignants, secouristes) ne sont pas suffisamment formés pour leur mission.

- Les décisions médicales sont influencées par les restrictions budgétaires (renoncement à la prescription de médicaments coûteux (par ex. traitement de l'hépatite C), à des mesures de prévention efficaces, à la vaccination [4]).

- Du fait que toutes les personnes détenues ne disposent pas d'une assurance de maladie, des divergences sont souvent constatées concernant le niveau de l'offre de soins.

\section{Garantie de l'indépendance}

Quelles que soient ses conditions particulières d'exercice, le médecin doit bénéficier d'une totale indépendance à l'égard des autorités policières ou pénitentiaires. Ses décisions cliniques et toutes autres évaluations relatives à la santé des personnes détenues ne peuvent être fondées que sur des critères strictement médicaux. Afin de garantir l'indépendance des médecins exerçant en milieu policier ou pénitentiaire, toute relation hiérarchique ou même contractuelle directe entre ces derniers et la direction de l'établissement doit être évitée à l'avenir (Directives de l'ASSM, chapitre 12).

La garantie de l'équivalence des soins et l'indépendance du médecin sont étroitement liées. Souvent les médecins (et les soignants) se retrouvent dans un conflit de loyauté entre leurs devoirs professionnels envers le patient et leurs devoirs - explicites ou implicites, réels ou supposés - envers des tiers. Les directives de l'ASSM «Exercice de la médecine auprès de personnes détenues» décrivent des situations typiques qui soulèvent des conflits de loyauté (par ex. situations d'expertise, traitement sous contrainte, grève de la faim, etc.). En particulier, 
le défaut ou l'insuffisance de ressources peuvent provoquer des situations délicates, par ex. lorsque des patients souffrant d'une maladie psychique sont détenus dans une institution qui n'est pas équipée pour de telles situations. Celles-ci peuvent déclencher des peurs au sein du personnel et les inciter à revendiquer des traitements injustifiés du point de vue médical (par ex. traitement sous contrainte). De même, les situations dans lesquelles les décisions médicales sont influencées par des réflexions économiques au sein de l'institution sont délicates. Lorsque les médecins sont directement employés par l'institution pénitentiaire ou les autorités judiciaires, la probabilité qu'ils soient influencés dans leurs décisions médicales est certainement plus élevée.

Dans ce contexte, la garantie de l'indépendance en milieu carcéral est primordiale. En Suisse, on constate cependant des profondes divergences quant à l'observation de ce principe. Alors que dans certaines institutions des règles formelles relatives à l'indépendance ont été instaurées, dans d'autres, elles sont insuffisantes, voire inexistantes.

L'expérience montre que, dans les pays où la médecine pénitentiaire ne dépend pas de l'autorité d'exécution, mais des autorités sanitaires, l'indépendance du médecin est renforcée et par conséquence la qualité des soins s'est améliorée [5]. Cette conclusion repose sur les facteurs suivants:

- Une meilleure continuité des soins grâce à une coopération entre les services de santé en prison et ceux pour les personnes libres;

- L'implication de l'institution pénitentiaire dans les campagnes de santé publique;

- Une amélioration de la surveillance épidémiologique et des mesures de prévention;

- Des améliorations en matière de recrutement, de motivation, de qualité et de prise en charge du personnel médical exerçant en milieu carcéral.

\section{Attentes de I'ASSM}

La mise en œuvre des principes fondamentaux décrits ci-dessus implique selon l'ASSM que les conditions suivantes soient remplies:

- Les infrastructures appropriées et les ressources nécessaires à l'équivalence de la prise en charge médicale doivent être à disposition. La prise en charge médicale doit être comprise globalement. Elle inclut les mesures diagnostiques, thérapeutiques et de soins, mais également des prestations médicales préventives, comme par ex. la vérification des vaccinations lors de l'examen d'entrée et, si nécessaire, la mise à jour des vaccins, la remise de drogues de substitution, etc. Dans l'idéal, des standards valables pour l'ensemble de la Suisse devraient être définis pour la prise en charge médicale, les conditions cadres et les ressources requises.

- Les tâches et les responsabilités de toutes les per- sonnes intervenant dans les mesures et les décisions concernant la santé de personnes détenues (médecins, personnel soignant, personnel pénitentiaire) doivent être clairement définies.

- L'indépendance professionnelle des médecins exerçant en milieu carcéral doit être garantie. Il convient de définir avec précision la procédure à suivre en cas de divergences d'opinion entre les professionnels de la santé et les autorités pénitentiaires pour les décisions concernant la santé des personnes détenues.

- Les différents groupes de collaborateurs actifs dans l'administration pénitentiaire doivent connaître les principes juridiques et éthiques en vigueur dans l'exercice de la médecine en milieu carcéral.

Le principe de l'équivalence des soins est aussi valable pendant la détention en vue d'un renvoi. Le déroulement des examens et des traitements médicaux selon les règles de l'art médical doit être garanti. Ceci concerne tout particulièrement l'évaluation de l'aptitude au transport de personnes détenues en vue d'un renvoi.

Le projet «Lutte contre les maladies infectieuses en milieu carcéral (LuMMic)» [6], lancé par la Conférence des directrices et directeurs des départements cantonaux de justice et police et les Offices fédéraux de la justice et de la santé publique, constitue une étape importante pour améliorer la situation dans le domaine de la médecine pénitentiaire. L'ASSM soutient dans toute leur étendue les recommandations de ce projet pour l'harmonisation de la prise en charge médicale dans l'exécution judiciaire suisse.

\section{Approuvée par la Commission Centrale d'Ethique de l'ASSM le 20 janvier 2012.}

\section{Références}

1 Sprumont D, Corpataux V. Traitement forcé en prison: analyse juridique d'un dérapage des autorités. Dans: Rapports entre médecins et autorités: indépendance ou collaboration? Actes de la $18^{\mathrm{e}}$ Journée de droit de la santé. Neuchâtel: Université de Neuchâtel; 2011. p. 111-38.

2 Comité des Ministres du Conseil de l'Europe: «Clinical decisions should be governed only by medical criteria. Health care personnel should operate with complete independence within the bounds of their qualifications and competence». Rec R(98)7; 1998.

3 Rieder JP. Rev Méd Suisse. 2010;28:1463.

Wolff H, Sebo P, Haller DM, Eytan A, Niveau G, Bertrand D, Getaz L, Cerutti B. 2011. Health problems among detainees in Switzerland: a study using the ICPC-2 classification. BMC Public Health. 2011;11:245. Gravier B, Iten A. Epidémiologie et prévention des infections dans les prisons de Suisse romande. Rapport au FNS. Lausanne; 2005.

4 Masia M, Achermann C, Richter M, Hostettler U. Auswertungsbericht zur Fragebogenerhebung 
«Analyse von Präventionsmassnahmen und Behandlungsangeboten von Infektionskrankheiten und Drogenabhängigkeit in Schweizer Anstalten des Freiheitsentzugs». 15. März; 2007.

Jurgens R, Ball A, Verster A. Interventions to reduce HIV transmission related to injecting drug use in prison. Lancet Infect Dis. 2009:9:57-66.

5 Elger BS. Towards Equivalent Health Care of Prisoners: European Soft Law and Public Health Policy in Geneva. Journal of Public Health Policy. 2008;29:192-206
6 Projet «Lutte contre les maladies infectieuses en milieu carcéral (BIG) 2008-2011» de la Conférence des directrices et directeurs des départements cantonaux de justice et police, de l'Office fédéral de la justice et de l'Office fédéral de la santé publique (www.bag.admin. ch/hiv_aids/05464/05484/05488/index. html?lang=de).

\section{Annexe aux directives médico-éthiques «Exercice de la médecine auprès de personnes détenues» \\ Conseils pratiques relatifs à l'application des directives}

Groupe de travail *

* Prof. Christian Kind, SaintGall, (Président) Dr Bidisha Chatterjee, Berne Dr Monique Gauthey, Genève Prof. Bruno Gravier, Prilly Prof. Samia Hurst, Genève Dr Fritz Ramseier, Brugg lic. iur. Michelle Salathé, ASSM Bâle

Anna Schneider Grünenfelder, Berne Prof. Dominique Sprumont, Neuchâtel

Marianne Wälti-Bolliger,

Delémont

Dr Hans Wolff, Genève

\section{Introduction}

En 2010, les gros titres de la presse relataient la prise en charge médicale d'un détenu en grève de la faim, une décision du Tribunal fédéral [1] et la participation de médecins aux renvois forcés. Ces actualités ont relancé les discussions au sein du corps médical [2] et incité l'Académie Suisse des Sciences Médicales (ASSM) à se pencher sur ce thème et à vérifier l'actualité et la praticabilité de ses directives médicoéthiques relatives à «L'exercice de la médecine auprès de personnes détenues», qui datent de 2002. La Commission Centrale d'Ethique (CCE) a chargé un groupe de travail de cette mission. Se fondant sur le rapport de ce groupe de travail, elle a conclu que ces directives, rédigées sur la base de documents internationaux mondialement reconnus, étaient toujours d'actualité. Toutefois, les expériences des médecins pénitentiaires montrent que les principes éthiques énoncés dans les directives sont encore insuffisamment appliqués dans l'exécution des peines et des mesures et que, dans certains domaines, il reste des efforts à faire dans ce sens. C'est pourquoi des conseils pratiques pour l'application des directives médico-éthiques relatives à «L'exercice de la médecine auprès de personnes détenues» ont été élaborés; ceux-ci constituent une annexe aux directives. Par ailleurs, alors que dans les directives de 2002 les soignants n'avaient été mentionnés qu'en marge, leur rôle est décrit plus précisément dans le présent document.

\section{Caractère contraignant des directives}

L'arrêt du tribunal fédéral dont il est question a déclenché un débat juridique sur le poids des directives médico-éthiques de l'ASSM. Un médecin pénitentiaire peut-il invoquer les directives, lorsqu'il reçoit un ordre qui transgresse les principes éthiques ou est-il tenu d'exécuter dans tous les cas les décisions de la direction de l'établissement pénitentiaire ou des autorités judiciaires?

Comme toutes les directives de l'ASSM, celles qui traitent de «L'exercice de la médecine auprès de personnes détenues» s'adressent aux médecins et autres professionnels de la santé; leur but est d'apporter une aide à la pratique médicale quotidienne. Il ne s'agit pas de droit impératif, mais le caractère contraignant peut découler du contrat avec le patient ou de l'adhésion par un médecin à une association. La FMH ayant intégré les directives dans leur code déontologique, cellesci sont obligatoires pour tous les membres de la FMH. Mais les directives ont également des conséquences légales en-dehors de ce cadre. En effet, le Tribunal fédéral leur reconnait dans une jurisprudence constante la valeur de règles de l'art médical. Le juge peut s'appuyer sur les directives afin de déterminer la diligence d'un médecin dans un cas particulier. Par les biais des règles de responsabilité, les directives servent ainsi de référence pour juger si un médecin n'a pas violé ses obligations par non-respect des standards professionnels.

Par conséquent les règles matérielles consacrées dans les directives de l'ASSM sont particulièrement 
importantes. Les directives médico-éthiques relatives à «L'exercice de la médecine auprès de personnes détenues», publiées en 2002 , récapitulent de façon aisément compréhensible les droits fondamentaux valables en médecine pénitentiaire et offrent ainsi une orientation pour la pratique.

En premier lieu, le principe de l'équivalence des soins pour les personnes en détention et celles en liberté (chapitre 5) est explicitement reconnu dans la jurisprudence de la Cour européenne des droits de l'homme et en droit cantonal, comme en Valais ou à Genève. De même, l'indépendance des médecins dans le choix et la fourniture de soins, exigée dans les directives (chapitre 12), repose directement sur les principes de base en matière de droit des patients et d'obligations professionnelles. On notera ainsi que les articles 56ss du Code pénal suisse précisent à quelles conditions, en se basant sur l'avis d'expert d'un médecin, le juge devra renoncer à prononcer une peine pour lui substituer une mesure thérapeutique lorsqu'un détenu souffre de troubles de santé et, de la même manière, comment le juge devra s'en remettre à l'avis du médecin pour déterminer la nature et les conditions de réalisation de cette mesure dans un établissement approprié. A chaque étape, le Code pénal définit le champ de compétence propre du juge et du médecin en garantissant à ce dernier son indépendance (cf. chapitre 4 ci-après). Ainsi, lorsque le juge fait référence à l'avis du médecin, il en accepte les règles et les conditions de pratique.

\section{Application du droit à l'équivalence de traitement}

Chapitre 5 des directives «Exercice de la médecine auprès de personnes détenues»

Le droit à l'équivalence de traitement est un principe fondamental en médecine pénitentiaire. A l'exception d'un libre choix plus limité du médecin, les personnes détenues ont, en matière de santé, les mêmes droits que les autres patients. Non seulement les directions des établissements pénitentiaires, mais aussi tous les médecins et soignants impliqués dans la prise en charge du patient sont tenus de respecter ce droit.

Le droit à l'équivalence de traitement n'englobe pas seulement l'accès aux mesures de santé préventives, diagnostiques, thérapeutiques et de soins, mais également les règles de base de la relation médecinpatient, comme par exemple le droit à l'autodétermination et à l'information et le respect de la confidentialité. A ce propos, les médecins qui interviennent en milieu carcéral sont tenus au secret professionnel consacré par l'article 321 CPS et ne peuvent dévoiler des informations concernant leurs patients à des tiers en dehors du cadre étroit fixé par la loi (cf. chapitre 10 des directives).

Ceci devient problématique dans ce contexte, lorsque, par manque de ressources, le personnel de surveillance est chargé de la distribution de médicaments. Si les médicaments sont délivrés par des per- sonnes autres que le personnel médical qualifié, cela ne constitue pas seulement une atteinte au secret professionnel, mais aussi une infraction à la loi sur les produits thérapeutiques. En effet, celle-ci stipule que les personnes habilitées à distribuer les médicaments doivent être définies avec précision, sans aucune exception pour le régime pénitentiaire. Sont autorisés à délivrer des médicaments les pharmaciens et autres personnes exerçant une profession médicale universitaire, à savoir les médecins, dentistes, vétérinaires et chiropraticiens, ainsi que tout autre professionnel de la santé dûment formé, sous le contrôle d'un représentant des professions médicales universitaires susmentionnées, et au bénéfice d'une autorisation des autorités sanitaires cantonales compétentes, généralement le pharmacien cantonal.

Face à la réalité de la prise en charge médicale et des soins dans les prisons suisses, il est indispensable de trouver des solutions qui permettent d'une part un fonctionnement optimal de la prise en charge médicale avec des possibilités de délégation acceptables tout en respectant, d'autre part, les dispositions légales. Lorsque des personnes non habilitées délivrent des médicaments, les conditions suivantes doivent être observées:

1. Le médicament fait l'objet d'une prescription médicale.

2. Il a été livré à l'établissement par une pharmacie publique, les médicaments stockés dans l'établissement étant placés sous le contrôle et la responsabilité d'un pharmacien autorisé.

3. Dans la mesure du possible, les médicaments sont remis sous une forme neutre (semainiers ou barquettes) qui permettent de respecter la confidentialité.

4. Le surveillant se limite à vérifier que les barquettes soient correctement distribuées. En cas de doute, il doit contacter le pharmacien ou le médecin responsable et suivre ses instructions.

\section{Missions du médecin dans un établisse- ment d'exécution des mesures}

Chapitre 6 des directives «Exercice de la médecine auprès de personnes détenues»

La prise en charge de détenus dans le cadre d'une mesure judiciaire (art. 63 et 59 du Code pénal) fait partie des missions principales de la médecine pénitentiaire. L'ordonnance judiciaire pour l'exécution des mesures est basée sur une expertise psychiatrique. L'application d'une mesure doit être adéquate, proportionnelle et applicable (art. 56 CPS). Suivant les tribunaux, le dispositif thérapeutique préconisé est plus ou moins détaillé dans le jugement. La plupart du temps, il reprend de manière générale les termes de l'expertise psychiatrique. Se pose alors la question de la marge de manœuvre dont dispose le médecin à qui est confié le mandat par l'autorité pénale ou pénitentiaire. 


\section{L'acceptation du mandat de soins}

Un médecin ne doit accepter un mandat de soins que s'il a les compétences requises pour en remplir les objectifs. Ainsi, par exemple, il ne devrait accepter de prodiguer un suivi psychothérapeutique que s'il dispose des connaissances spécifiques et de l'expérience pratique pour soigner la personne détenue qui lui est confiée, sinon il doit refuser le mandat.

Avant d'accepter un mandat, le médecin doit être conscient de ce que celui-ci signifie pour luimême et pour le patient. Il doit notamment s'assurer que le patient est prêt à le délier du secret professionnel pour qu'il puisse transmettre aux autorités compétentes les informations indispensables au contrôle du déroulement de la mesure thérapeutique. Dans l'idéal, les modalités de la rencontre thérapeutique dans ce contexte (déliement du secret médical, etc.) devraient être définies au préalable.

\section{L'évaluation de l'état du patient et des options thérapeutiques}

Le médecin doit procéder à une anamnèse minutieuse, évaluer les options thérapeutiques, élaborer une proposition de traitement, la discuter avec le patient et obtenir son consentement. Le choix du traitement est basé exclusivement sur des considérations médicales, il s'agit donc d'une décision purement médicale. Il n'est pas rare que des conflits surgissent dans ces situations. Par exemple, lorsqu'un patient souhaite un traitement pharmacologique visant à inhiber ses pulsions sexuelles dans le but d'obtenir un élargissement de la part des autorités, le médecin ne peut soutenir la demande que si, du point de vue médical, le traitement est pertinent. Le traitement pharmacologique doit être efficace et il ne doit présenter aucune contre-indication. Le fait que des options thérapeutiques soient mentionnées dans le jugement conduisant au prononcé de la mesure ne dispense pas le médecin d'observer les prescriptions éthiques valables pour tout traitement.

Il est important de garder à l'esprit que la mesure pénale se limite à fixer le cadre de l'exécution d'une décision de justice. Néanmoins, à l'intérieur de ce cadre, le médecin conserve toutes ses prérogatives pour conduire un traitement selon l'appréciation médicale exclusive qu'il peut faire de la situation. Il lui appartiendra de rendre compte à l'autorité judiciaire de la manière dont il a exécuté ou non le mandat qui lui a été confié, sans perdre de vue qu'il reste tenu par une obligation de moyen et non par une obligation de résultat.

\section{Application de traitements sous contrainte}

Chapitre 7 des directives «Exercice de la médecine auprès de personnes détenues»

Les dispositifs de contrainte dans le cadre thérapeutique peuvent revêtir différentes modalités: il peut s'agir de dispositifs de contention physique, de séda- tions médicamenteuses ou de traitements pharmacologiques, psychotropes la plupart du temps, imposés sans le consentement du patient. Des traitements sous la contrainte ne peuvent être appliqués que dans les situations d'urgence décrites au chapitre 7.3. selon les mêmes critères valables pour des personnes non détenues. Ils doivent toujours être indiqués et prescrits par un médecin. Lors de leur application, la dignité du patient doit être respectée et les mesures doivent être adéquates et proportionnelles. C'est le rôle du médecin de poser l'indication et de veiller au respect des bases juridiques. Les médecins et infirmiers ne peuvent pas introduire des mesures de contrainte sur ordre des autorités.

Les mesures de contrainte peuvent être traumatisantes non seulement pour le patient, mais également pour l'équipe soignante; de ce fait, la décision devrait être - dans toute la mesure du possible - discutée et soutenue par toute l'équipe (médecins, infirmiers et personnel de sécurité).

\section{Procédure en cas de grève de la faim}

Chapitre 9 des directives «Exercice de la médecine auprès de personnes détenues»

Les médecins et soignants exerçant en milieu pénitentiaire sont régulièrement confrontés à des détenus en grève de la faim. Celle-ci doit être comprise comme un acte de protestation - souvent ultime d'une personne qui ne se sent plus en mesure d'être entendue d'une autre façon. Le gréviste de la faim ne veut pas mourir; il veut avant tout que sa revendication aboutisse. Il sait qu'une issue fatale est possible si la situation se dégrade au point de devenir un conflit insoluble.

Lors du choix de la procédure adéquate, il est important de bien distinguer les différentes situations dans lesquelles une alimentation artificielle (au moyen d'une sonde gastrique ou d'une perfusion) est évoquée chez un gréviste de la faim:

1. La personne détenue est capable de discernement, elle décline l'alimentation artificielle et sa vie n'est pas en danger immédiat. Une alimentation forcée dans cette situation a été qualifiée de torture par la Cour Européenne de Justice.

2. La personne détenue est capable de discernement, elle décline l'alimentation forcée et la poursuite de la grève de la faim met sa vie en danger.

3. La personne détenue est devenue incapable de discernement suite à la grève de la faim, elle a consigné son opposition à l'alimentation artificielle dans des directives anticipées valides et le renoncement à l'alimentation artificielle signifie qu'elle encourt un danger de mort imminent.

4. La personne détenue est incapable de discernement (ou bien à la suite de la grève de la faim ou d'une autre cause), elle ne dispose pas de directives anticipées valides, qui interdisent une alimentation artificielle dans cette situation, et le 
renoncement à l'alimentation artificielle signifie qu'elle encourt un danger de mort imminent. Selon les directives de l'ASSM, du point de vue médical, une alimentation artificielle n'est indiquée que dans la situation 4 et peut être administrée sans emploi de force dans la majorité des cas. Dans les autres situations, elle serait en contradiction avec les directives et les règles de l'art médical.

Les directives de l'Académie Suisse des Sciences Médicales (ASSM) décrivent les missions des médecins en cas de grèves de la faim dans le chapitre 9. A titre complémentaire, les recommandations suivantes sont à observer:

- Les grévistes de la faim doivent bénéficier d'une évaluation médicale le plus rapidement possible (<24 h). Il convient de clarifier s'ils refusent seulement de se nourrir ou aussi de s'hydrater. Par ailleurs, les comorbidités (par ex. diabète, maladie psychiatrique ou insuffisance rénale) doivent être clarifiées. Par la suite, l'état du patient doit être évalué quotidiennement par un professionnel de santé.

- Le gréviste de la faim doit être informé des conséquences d'un jeûne prolongé sur son état physique et psychique. Les risques et les mesures de protection (apport de liquide, substitution de vitamines et d'électrolytes) de même que les risques liés à la réintroduction de l'alimentation («refeeding-syndrome») doivent être discutés (chapitre 9.1.).

- De la nourriture doit être proposée quotidiennement au gréviste de la faim (chapitre 9.5.).

- La relation de confiance est la base de toute thérapie. Dès lors il est important de clarifier les rôles et de garantir que le médecin et les infirmiers agissent indépendamment des autorités non médicales. Il faut, en outre, garantir à la personne en grève de la faim que le médecin/soignant observe les directives de l'ASSM relatives à la prise en charge de personnes détenues, notamment en matière de confidentialité et de droit à l'autodétermination et à l'intégrité corporelle.

- Il convient également de s'assurer que la personne détenue a décidé librement de la grève de la faim, sans pression extérieure.

- La capacité de discernement doit être évaluée régulièrement (au moins chaque semaine), le cas échéant par des experts indépendants.

- Au plus tard, lorsqu'une hospitalisation est envisagée, il est souhaitable de proposer au patient de rédiger des directives anticipées.

\section{Obligations lors des procédures d'expulsion du territoire suisse}

Chapitre 6 des directives "Exercice de la médecine auprès de personnes détenues"

Le principe de l'équivalence des soins est aussi valable pendant la détention en vue d'un renvoi. Il implique l'obligation de procéder aux examens et traitements médicaux dans les règles de l'art. (cf. chapitre 3). Les obligations liées aux renvois sont décrites en détail dans les directives de l'ASSM (chapitre 6). Elles stipulent notamment que le médecin ne peut assumer la responsabilité de la mesure et doit refuser de prêter son concours s'il est persuadé que les moyens utilisés font courir un risque de santé immédiat et majeur au patient.

Il arrive toujours que des personnes qui s'opposent à un renvoi soient ligotées au moyen d'attache-câbles (renvoi de niveau IV). Conformément à la directive sur le retour de l'UE [3], depuis le $1^{\text {er }}$ janvier 2011, un observateur indépendant doit surveiller le renvoi. Elle prévoit également qu'un médecin (disposant d'une formation de médecin urgentiste), et le cas échéant un secouriste, garantissent l'assistance médicale de la personne détenue. Le ligotage rend toutefois une évaluation clinique plus difficile. Cette difficulté est aggravée par le fait que dans la plupart des cas, les actes ou les examens médicaux préalables ont été insuffisants, car pendant la détention en vue d'un renvoi seules les interventions urgentes sont remboursées. L'évaluation de l'aptitude au transport des détenus en vue du renvoi et l'assistance médicale pendant le renvoi font partie des devoirs du médecin et doivent être réalisées dans les règles de l'art. Par contre, dans des conditions qui entravent ou empêchent l'évaluation ou la prise en charge médicale, le médecin a le devoir moral et juridique de refuser d'être associé au renvoi.

\section{Approuvée par la Commission Centrale d'Ethique de l'ASSM le 20 janvier 2012.}

\section{Références}

1 Arrêt du Tribunal fédéral du 26 août 2010 dans l'affaire B. Rappaz.

2 Fédération des médecins suisses FMH, Association Suisse des Infirmières et Infirmiers ASI, Académie Suisse des Sciences Médicales ASSM, Commission Centrale d'Ethique de l'Académie Suisse des Sciences Médicales CCE, Conférence suisse des médecins pénitentiaires, Forum du personnel soignant des établissements de détention en Suisse et 74 signataires individuels. Grève de la faim en prison - A propos de l'arrêt du Tribunal fédéral du 26 août 2010. Bull Méd Suisses. 2010;91(39):1518-20.

3 Directive sur le retour (directive 2008/115/CE).

\section{Annexe}

\section{Documents de référence}

United Nations:

- Body of Principles for the Protection of All Persons under Any Form of Detention or Imprisonment. Adopted 1989. www.2.ohcr.org/english/law/ bodyprinciples.htm

- Istanbul Protocol Manual on the Effective Investigation and Documentation of Torture and Other Cruel, Inhuman or Degrading Treatment or Punishment; 1999. www.ohchr.org/Documents/ Publications/training8Rev1en.pdf 
- Standard Minimum Rules for the Treatment of Prisoners. Adopted 1955. www2.ohchr.org/english/ law/treatmentprisoners.htm

- Resolution 37/194, 1982: Principles of Medical Ethics relevant to the role of health personnel, particularly physicians, in the protection of prisoners and detainees against torture, and other cruel, inhuman or degrading treatment or punishment. Adopted 1982. www.cirp.org/library/ethics/UN-medical-ethics

\section{Council of Europe:}

- Recommendation Rec(2006)2 on the European Prison Rules. wcd.coe.int/ViewDoc.jsp?id=955747

- Recommendation R(98)7 of the Committee of Ministers to member states on the ethical and organisational aspects of health care in prison. Council of Europe Publishing. Strasbourg: 1999. wcd. coe.int/com.instranet.InstraServlet?command=com. instranet. CmdBlobGet\&InstranetImage $=530653 \&$ Sec Mode $=1 \&$ DocId $=459562 \&$ Usage $=2$

European Committee for the Prevention of Torture and Inhuman or Degrading Treatment or Punishment, (CPT):

- The CPT standards. CPT/Inf/E (2002)1-Rev.2009. www.cpt.coe.int/en/documents/eng-standards.pdf

\section{World Medical Association:}

- Declaration of Tokyo. Guidelines for Physicians Concerning Torture and Other Cruel, Inhuman or Degrading Treatment or Punishment in Relation to Detention and Imprisonment. Tokyo 1975 revised Divonee-les-Bains 2005. www.wma.net/e/policy/c18. htm

- Declaration on Hunger Strikers. Malta 1991, revised Marbella 1992, revised Pilanesberg 2006 www.wma. net/e/policy/h31.htm

- Statement on Body Searches of Prisoners. Budapest 1993, revised Divonee-les-Bains 2005. www.wma. net/e/policy/b5.htm

- Declaration Concerning Support for Medical Doctors Refusing to Participate in, or to Condone, the Use of Torture or Other Forms of Cruel, Inhuman or Degrading Treatment. Hamburg; 1997. www.wma. net/e/policy/c19.htm

- Resolution on the Responsibility of Physicians in the Denunciation of Acts of Torture or Cruel or Inhuman or Degrading Treatment of Which They Are Aware. Helsinki; 2003. www.wma.net/e/policy/t1.htm

Conseil international des Infirmières (International

Council of Nurses) (ICN):

- Nurses' role in the care of detainees and prisoners. Adopted 1998, revised 2006. www.icn.ch/images/ stories/documents/publications/position_statements/ A13_Nurses_Role_Detainees_Prisoners.pdf

- Ethikkodex. 13.11.2009. www.icn.ch/about-icn/codeof-ethics-for-nurses/

\section{Littérature}

- Binswanger IA, Krueger PM, Steiner JF. Prevalence of chronic medical conditions among jail and prison inmates in the USA compared with the general population. J Epidemiol Community Health. 2009;63:912-9.

- Fazel S, Baillargeon J. The health of prisoners. Lancet. 2011.377:956-65

- Fazel S. Danesh J. Serious mental disorder in 23000 prisoners: a systematic review of 62 surveys. Lancet. 2002;359:545-50.

- Gravier B, Iten A. Epidémiologie et prévention des infections dans les prisons de Suisse romande. Rapport au FNS. 2005; Lausanne.

- Harding T W. La santé en milieu carcéral. Bulletin d'information pénitentiaire. 1987;10:10-2.

- Jurgens R, Ball A, Verster A. Interventions to reduce HIV transmission related to injecting drug use in prison. Lancet Infect Dis. 2009;9:57-66.

- Kind C. Die Richtlinien der Schweizerischen Akademie der Medizinischen Wissenschaften zur Ausübung der ärztlichen Tätigkeit bei inhaftierten Personen - wo stehen wir heute? In: Guillod O, Sprumont D (Hrsg.). Rapports entre médecins et autorités: indépendance ou collaboration? 2011; S. $153 \mathrm{ff}$.

- Rüetschi D. Ärztliches Standesrecht in der Schweiz - Die Bedeutung der Medizin-ethischen Richtlinien der Schweizerischen Akademie der Medizinischen Wissenschaften. In: Die Privatisierung des Privatrechts rechtliche Gestaltung ohne staatlichen Zwang. Jahrbuch Junger Zivilrechtswissenschaftler. 2002; S. 231-55.

- Sprumont D, Schaffter G, Hostettler U, Richter M, Perrenoud J. Pratique médicale en milieu de détention. Effectivité des directives de l'Académie Suisse des Sciences Médicales sur l'Exercice de la Médecine auprès de Personnes Détenues. Mai 2009. www.unine.ch/ids/

- Wolff HP, Sebo DM, Haller A, Eytan G, Niveau D Bertrand, et al. Health problems among detainees in Switzerland: a study using the ICPC-2 classification BMC Public Health. 2011;11:245. 